\title{
Diversity of Avifauna from Dandoba Hill Forest and its Surrounding Areas (Dist - Sangli)
}

\author{
S. B. More \\ Department of Zoology, P. V. P. Mahavidyalaya, Kavathe Mahankal, Dist.- Sangli, (MS), India
}

\begin{abstract}
The survey of avifauna was carried out from the Dandoba forest and the nearby area of the forest. Dandoba is situated in Sangli district and on the boundary of Miraj and Kavathe Mahankal tahsil. This area is of low rainfall, with hills and slopes with some bushy plants, grass and trees. The study was carried out for three years. The villages nearby the Dandoba are largely agricultural. The principal crops taken being jawar, groundnut, sugarcane and tobacco. On a smaller scale bananas are also grown. Birds play important role in biodiversity by keeping insect pest under control. Over 364 hectors of land is under well irrigation which play very important role to maintain the bird diversity This region shows good number of birds during the month of September to December. This is because of availability of food and breeding ground. About 95 species belonging to 48 families were recorded from the study area.
\end{abstract}

Keywords: Dandoba. Avifauna and Diversity

\section{Introduction}

A bird has been described as a Feathered Biped. They are vertebrates, warm blooded animals (homeotherms). To assist in maintaining an even temperature, the body of a bird is covered with non-conducting feathers. Birds enjoy a wide distribution on the earth than any other class of animal. Birds are useful to human community such as destroyers of insect pests, as destroys of other vermin, as scavengers, as flower pollination agents, as seed dispersers, as food for man, feathers in cottage industry, and as fertilizer. [10]. A comparative study of birds reveal that there are recognizable differences in size, coloration and other details in those species which range over a wide area live under diversified natural conditions.

The forest type of Dandoba is tropical dry deciduous and thorny, scrubby forest. The vegetation area is hilly with slopes and plateaus. The main source of water is Bhose Talav, which supports flora and fauna. The main crop of this region is Tobbaco, Sugarcane, groundnut, chillies and cereals. This region is mainly dominated by different plant species such as Acacia, Azadiracta, Tectona, Albizzia, Bamboo, Erythrina, Beautia, Terminalia, Pithocolombia,
Eucalyptus, Lantena, Mangifera, Morinda, Tamarindus, Ficus, and with different weeds. Hence support sustainable place for Avifauna. Study Area of Dandoba belongs to Sangli district and on the boundary of Tahcil Miraj and Kavathae Mahankal having moderate rainfall of average $500 \mathrm{~mm}$. The climate is hot. Maximum temperature reaches up to $45^{\circ} \mathrm{c}$ in summer, while minimum goes down up to $19^{0} \mathrm{C}$.

\section{Methods and Materials}

The Taxonomic list of avifauna was recorded during the period of April 2012 to March 2015. The survey was carried out every month of year. The habitat, behavior of birds and movements and identification marks was observed by using binocular (Olympus 8- 16x4) zoom DPS 1. For identification referred, The book of Indian Birds ${ }^{\text {ec }}$ by Salim Ali, A Field Guide to the Birds of India by Krys Kazmierczak, Birds by Herbert S. Zim, ,Birds of the Indian Subcontinent ${ }^{e c}$ Richard Grimmett et.al.[1] [,2] ,[3], [7], [8], [9], [10], [11],[12]. Diversity of Avifauna from Dandoba Hill Forest and its Surrounding Areas. Resident of local (R), Migrant (M), Endangered (E) and Local Migrant (LM)

Scientific Name
1 FAMILY - Accpitridae
i) Pemis Ptilorhyncus
ii) Haliastur Indus
iii) Milvus migrans
iv) Accipeter badius
v) Butastur teesa
vi) Spizaetus limnaeetus
vii) Aquila rapax
viii) Ceryle rudis
ix) Halcyon smymensis
x) Alcedo atthis
2 FAMILY -Aegithinidae
i) Aegithiniatiphia
3 FAMLY -Alaudidae
i) Eremopteriex griseos
ii) Galerida malabarica

\section{Common Name}

Oriental honey buzzard

Brahminy kite

Indus Black kite

Shikra

White eyed buzzerd

Changeable hawk eagle

Tawny eagle

Pied kingfisher

White throated kingfisher

Common kingfisher

Common iora

Ashy crowned finch lark

Malabar creasted lark

\section{Status}

$\mathrm{R}$

$\mathrm{R}$

$\mathrm{R}$

$\mathrm{R}$

$R$

$\mathrm{R}$

LM

$\mathrm{R}$

$\mathrm{R}$

$\mathrm{R}$

R

$\mathrm{R}$

$\mathrm{R}$ 
5 FAMILY -Anatidae
i) Dendrocygna javanica
ii) Anas crecca
iii) Anas poecilorhyncha
iv) Anas clypeata
v) Aytha ferina

6 FAMILY- Ardeidae
i) Egretta alba
ii) Egretta intermedia
iii) Egretta garzetta
iv) Bubulcus coromandus
v) Ardea cinerea
vi) Ardeola grayii

7 FAMILY-Bucerotidae

i) Ocyceros birostris

8 FAMILY-Capitonidae i) Megalaima haemacephala

9 FAMILY -Campephagidae
i) Pericrocotus cinnamomeous

ii) Tephrodornis pondicerianus

10 FAMILY-Charadriidae
i) Vanellus indicus
ii) Charadius dubius
iii) Vanellus malabaricus

11 FAMILY -Ciconiidae

i) Ciconia episcopus

iii) Mycteria leucocephala

12 FAMILY -Cisticolidae
i) Prinia socialis
ii) Prinia inornata
iii) Orthotomus sutorius
iv) Prinia hodgsonii

13 FAMIIY-Columbidae

i) Streptopelia chinesis

ii) Columbo livia

14 FAMILY-Corcaciidae

i) Coracias benghalensis

15 FAMILY-Corvidae

i) corvus splendens

16 FAMILY -Cuculidae

i) Cacomantis passerinus

ii) Eudynamys scolopaceous

\section{FAMILY-Dicruridae}

i) Dicrurus leucophaeus
Lesser whitling duck $\quad \mathrm{M}$

Common teal M

Spotbill duck R

Northenshoveler M

Common pochard M

$\begin{array}{ll}\text { Great egret } & \text { R } \\ \text { Intermediate egret } & R \\ \text { Little egret } & R \\ \text { Cattle egret } & R \\ \text { Grey heron } & R \\ \text { Indian pond heron } & R\end{array}$

Indian grey hombill $\quad$ R

Copersmith barbet $\quad R$

Small minivet $\quad \mathrm{R}$

Common wood shrike $\quad \mathrm{R}$

Red wattled lapwing $\quad R$

Little ringed plover $R$

Yellow wattled lapwing $\quad R$

White necked stork R

Painted stork E

Ashy priania $\quad \mathrm{R}$

Plain prinia $\quad R$

Common tailor bird $\mathrm{R}$

Grey breasted prinia $\quad$ R

Spoted dove $R$

Rock pigeon $R$

Indian roller $\quad \mathrm{R}$

House crow $\quad \mathrm{R}$

Grey billed cuckoo LM

Asian koel $\mathrm{R}$

Black drongo $\quad \mathrm{R}$ 
18 FAMILY-Dicaeidae

i) Dicaeum erythrorhynchos

ii) Dicaeum agile

19 FAMILY -Hirundinidae
i) Hirundo smithii

ii) Hirund daurica

20 FAMILY- Laniidae
i) Lanius schach

ii) Lanius cristatus

21 FAMILY-Emberizidae

i) Emberiza melanocephala

ii) Melophus lathami

22 FAMILY-Estrildidae

i) Lonchura punctulata

23 FAMILY- Glareolidae

i) Cursonius coromandelicus

\section{FAMILY -Muscicapidae}
i) Copsychus saularis
ii) Luscinia
iii) Saxicola torquatus
iv) Cyornis tickelliae

25 FAMILY-Meropidae

i) Merops orientalis

26 FAMILY-Monarchidae

i) Terpsiphone paradisi

27 FAMILY -Motacillidae
i) Motacilla alba
ii) Motacilla cinerea
iii) Motacilla flava
iv) Anthus rufulus

28 FAMILY -Nectariniidae

i) Leptocoma zeylonica

ii) Cinnyris asiaticus

29 FAMILY-Paridae

i) Parus major

30 FAMILY --Passeridae

i) Passer domesticus

31 FAMILY -Pandionidae

i) Pandion halietus

32 FAMILY -Ploceidae

i) Ploceus philippinus

33 FAMILY -Phasianida

i) Pavo cristatus I

34 FAMILY-Phalacrocoracida

i) Phalacrocorax carbo

ii) Phalacrocora xniger

35 FAMILY Picidae

i) Dendrocopos mahrattensi

36 FAMILY- Psittacidae

i) Psittaculan cyanocephala

ii) Psittacula krameri

37 FAMILY- Pycnotidae

i) Pycnonotus luteolus

ii) Pycnonotus cafer
Thick billed flower picker

$\mathrm{R}$

Pale billed flowerpicker

$\mathrm{R}$

Wire tailed swallow

$\mathrm{R}$

Red numped swallow ?

$\mathrm{R}$

Long tailed shrike

Brown shrik

R

M

Black headed bunting

M

Crested bunting

$\mathrm{R}$

Scaly breasted munia

R

Indian courser

$\mathrm{R}$

Oriental magpie robin

brunnea Indian robin

Common stone chat

Tickells blue flycatcher

$\mathrm{R}$

$\mathrm{R}$

$\mathrm{M}$

R

Green bee eater

R

Asian paradise flycatcher

LM

White wagtail

Grey wagtail

Yellow wagtail

paddy field pipit

$\mathrm{M}$

$\mathrm{M}$

$\mathrm{M}$

R

Purple rumped sunbird

Purple sunbird

R

R

Greate tit

R

House sparrow

R

Osprey

M

Baya weaver

R

Indian peafowl

R

Great cormorant

R

Little cormorant

$\mathrm{R}$

Yellow crowned woodpecker

R

plum headed parakeet

R

Rose ringed parakeet

$\mathrm{R}$

White browed bulbul

R

Red vented bulbul 
International Journal of Science and Research (IJSR)

ISSN (Online): 2319-7064

Index Copernicus Value (2013): 6.14 | Impact Factor (2014): 5.611

38 FAMIIL -Rallidae

i) Amaurornis phoenicurus

ii) Fulica atra

39 FAMILY-Rhipiduridae

i) Rhipidura albicoilis

40 FAMILY-Scolopacidae

i) Actitis hypoleucos

41 FAMILY-Sternidae

i) Sterna aurantia

42 FAMILY- Sturnidae

i) Temenuchus pagodarum

ii) Acridotheres tristis

43 FAMILY -Threskiornithidae

i) Platalea leucorodia

ii) Threskiornis melanocephalus

iii) Pseudibis papillosa

44 FAMILY -Turnicidae

i) Tumix suscitator

45 FAMILY-Timaliidae

i) Chrysomma sinenes

46 FAMILY-Tytonidae

i) Tyto alba

47 FAMILY-Upupidae

i) Upupa epops

48 FAMILY-Zosteropidae

i) Zosteropspal pebrosus

\section{Results and Discussions}

The following taxonomic list was recorded from Dandoba Hill Forest and nearby areas. including Bhose Water reservoir. At least 95 birds genera and species belonging to 48 Families are recorded. This survey shows that there is wider range of major species in the Avifauna of Dandoba Hill Forest.and its surrounding areas. There are three different types of birds identified such as Resident of local (R), Migrant (M), Endangered (E) and Local Migrant (LM).

The percentage of - Resident - $0.67 \%$, Migrant - $0.21 \%$, Endangered - 0.03\%, and Local migrant - 0.07\%. This proves that this region supports good number of birds because of availability of different food items, shelter, resting grounds as well as peaceful and protective land. It is obvious that resident and local migrant birds dominate the avifauna in this region. In certain places a mosaic type of distribution were observed because of deforestation and water resources. After going to the checklist it is very clear that both terrestrial (land) and aquatic birds from Dandoba and its surrounding areas represents 48 Families, Genera and Species 95, counted as a rich Biodiversity. Some species are endangered and frequently visit to this region because of natural resources available in terms of shelter, food and breeding grounds. Near feature Dandoba region has to be protected.
White breasted water hen

Common coot

R

LM

White throated fantail

R

Common Sandpiper

$\mathrm{R}$

River tem

$\mathrm{R}$

$\begin{array}{ll}\begin{array}{l}\text { Brahminy starling } \\ \text { Common Myna }\end{array} & \begin{array}{l}\text { R } \\ \text { Eurassian spoonbill }\end{array} \\ \begin{array}{l}\text { Black headed ibis } \\ \text { Black ibis }\end{array} & \text { E } \\ \text { Barred botton quill } & \text { R } \\ \text { Yellow eyed babbler } & \text { R } \\ \text { Bam owl } & \text { R } \\ \text { Common hoopoe } & \text { R } \\ \text { Oriental white eye } & \text { R }\end{array}$

\section{Acknowledgment}

Author thanks to Prof V. A. Sawant, former head Department of Zoology, Shivaji Universiy Kolhapur for his encouragements.

\section{References}

[1] Ali, S. and Whisteler, H. (1933a): The Hyderabad State Ornithology Survey Part 3.J. Bombay Nat. Hist., Soci.,36(2):356-390.

[2] Ali, S. and Reply S.D. (1983b): A pictoral Guide to the birds of the Indian Subcontinent Bom. Nat. Hist. Soc.Mumbai.

[3] Ali, S. and Ripley, S.D. (1974): Hand book Of India and Pakistan. Oxford University Press. [ISSN 0975 6272]

[4] Ali, S. and Ripley, E. (1987): Compact hand book of Birds and adjacent Countries.Oxford University Press.Koskimies, P. (1987): Monitoring of finish bird Fauna Birds as Environmental Indicator (in finish with English summery) Ministry Environment Ser. A49. 1 255.

[5] Majumdar, N. (1984): On a Collection of Birds from Adilabad Dist. Andhra Pradesh. Ripley, S. D., B. M. Beehikar and Krishnaa

[6] K.S.R. Raju (1987): Birds of VishakapattanWhatsAndra Pradesh. J.Bom.Nat. His. Soc. 84(3): 540-559.

[7] Krys Kazmierczak : A Field Guide to the Birds of India 


\section{International Journal of Science and Research (IJSR) \\ ISSN (Online): 2319-7064}

Index Copernicus Value (2013): 6.14 | Impact Factor (2014): 5.611

[8] Patil S.R. et al. - Checklist of Avifauna from Lake Mayni, Dist- Stara , (M.S.), India During Oct-2005to Feb-2006 (Proceedings of National Workshop on Recent Trades in Biotechnology).

[9] Rinivasula, S. (2004): Birds of Kawal Wildlife Sanctuary, Andra Pradesh. India. J. of Bom. Nat. Soc. 101(1):3-25.

[10]Richard Grimmett, Tim Inskipp and Carol Inskipp : Birds of Indian Subcontinent,

[11] Salim Ali - The book of Indian birds

[12] Stenmetz (2003): Birds are overlooked top predators in aquatic food webs .Ecology, 84(5)1324-1328.

[13] Taher, S.A. and Pittie, A. (1994): Additions to "A Checklist of Birds of Andra Pradesh."'Mayur 11:1-5.

[14] Birding notes Pitta, A. (1987): Bom. Nat. Hist. Soc. Mumbai, Buceros 6(1):11-37.

[15] Champien, H. G. (1968): A revised survey of forest Types India. Govt. of India , New Delhi. 